\title{
Oil-soluble Ni-Mo sulfide nanoparticles and their hydrogenation catalytic properties
}

\author{
Liu Chenguang ${ }^{1}$, Wang Shutao ${ }^{1,2}$, An Changhua ${ }^{1,2 *}$, Yan Fang ${ }^{1}$, He Jie ${ }^{1}$, \\ Wang Zongxian ${ }^{1}$ and Zhang Qin-Hui ${ }^{\text {*** }}$
}

\author{
${ }^{1}$ State Key Laboratory of Heavy Oil Processing, College of Chemical Engineering, China University of Petroleum, Qingdao, \\ Shandong 266580, China \\ ${ }^{2}$ Key Laboratory of New Energy Physics \& Materials Science in Universities of Shandong, College of Science, China \\ University of Petroleum, Qingdao, Shandong 266580, China
}

(C) China University of Petroleum (Beijing) and Springer-Verlag Berlin Heidelberg 2013

\begin{abstract}
Oil-soluble bimetallic Ni-Mo sulfide nanoparticles (NiMoS) with narrow size distribution were successfully synthesized through a composite-surfactants-assisted- solvothermal process. The surface functionality and lipophilicity of the Ni-Mo sulfides were shown by transmission electronic microscopy, Fourier transform infrared and ultraviolet spectroscopy. The as-prepared Ni-Mo sulfides supported on activated carbon (NiMoS/AC) exhibited enhanced catalytic activity towards naphthalene hydrogenation instead of cracking. For comparison, $\mathrm{CoMoS} / \mathrm{AC}$ and $\mathrm{MoS}_{2} / \mathrm{AC}$ catalysts were also prepared through similar procedures, and it was found that their catalytic performance decreased in the order of NiMoS/ $\mathrm{AC}>\mathrm{CoMoS} / \mathrm{AC}>\mathrm{MoS}_{2} / \mathrm{AC}$. Furthermore, the activity of the bimetallic NiMoS nanocatalyst can be effectively tuned via variation of the atomic ratio of $\mathrm{Ni} /(\mathrm{Ni}+\mathrm{Mo})$.
\end{abstract}

Key words: Bimetallic sulfide, activated carbon, hydrogenation, nanocatalyst, naphthalene

\section{Introduction}

The search for highly efficient hydrogenation (HYD) catalysts for the petrochemical industry is of great significance for energy saving and environmental protection. Moreover, conversion of aromatic compounds during hydrotreatment to avoid their negative effects on the cetane numbers has received significant attention (Ferraz et al, 2010; He et al, 2011; 2013; Mendez et al, 2013; Temel et al, 2010). Particularly, catalysts based on molybdenum sulfides have attracted much interest for HYD reactions (Bellussi et al, 2013; Daage and Chianelli, 1994). The performance of a sulfide catalyst in the hydrofining process is closely related to its particle size and dispersion in nonaqueous media. Therefore, synthesis of a HYD catalyst with high surface area and improved oil-solubility, to eliminate particle aggregations, and to enhance the HYD performance, is an important issue.

Generally, there are two ways to obtain sulfides with improved catalytic efficiency. One is to load the sulfide components onto different supports, such as titania (Azizi et al, 2013; Kibsgaard et al, 2009), alumina (Ju et al, 2012; Lu et al, 2012; Si et al, 2010; Yao et al, 2012; Zhang et al, 2012), zirconia (Al-Daous and Ali, 2012; Jia et al, 2005), activated carbon (AC) (de la Puente et al, 1999; Liu et al,

*Corresponding author. email: anchh@upc.edu.cn

**Corresponding author. email: qhzhang@upc.edu.cn

Received July 11, 2013 2011a; Shi et al, 2010), and zeolites with different $\mathrm{Si} / \mathrm{Al}$ atomic ratios (Mendez et al, 2013; Yin et al, 2011). In such cases, the properties of the support, i.e., the porosity, surface area, acidity, and mechanical strength, will determine the characteristics and activities of the active sulfide phase, which is anchored on the support through the interactions of the sulfide phase and support at S-edge and metal-edge sites. Another method is to incorporate promoting elements, such as cobalt $(\mathrm{Co})$ or nickel $(\mathrm{Ni})$, into the sulfide nanoparticles (NPs) (Zhang et al, 2013; Zhou et al, 2012). The promoting elements of $\mathrm{Ni}$ or Co can weaken the edge anchoring of the active phase towards the supports. However, introduction of Co often encounters sintering and segregation (Eijsbouts et al, 2007; McCormick et al, 1989). Moreover, the Ni-Mo sulfides usually possess a high ratio of Brönsted to Lewis acid sites (Ferraz et al, 2010; Kibsgaard et al, 2009), which intensify the coordinative unsaturation of the transition metal atoms and their edge bonding effect (Kibsgaard et al, 2009; Yoosuk et $\mathrm{al}, 2010$ ). Therefore, incorporation of $\mathrm{Ni}$ is more preferred in terms of the stability, activity, and cost reduction of catalyst. The synergetic effect between the promoting elements and the catalysts can significantly improve the activity of the composite sulfide catalyst. Consequently, integration of the support effect and the promoting effect of transition metals would be highly promising for designing a novel HYD catalyst (Kibsgaard et al, 2009).

As part of our group's continuing efforts to develop efficient HYD nanocatalysts, this paper reports the synthesis 
of oil-soluble Ni-Mo sulfide (NiMoS) NPs with narrow size distribution through a composite-surfactants-assistedsolvothermal (CSAS) process. The as-produced bimetallic Ni-Mo sulfide was used as a precursor to prepare HYD nanocatalyst supported on activated carbon (AC). The catalytic HYD reactions were carried out with naphthalene (NAT, $\mathrm{C}_{10} \mathrm{H}_{10}$ ) as the model molecule of aromatics. For comparison, CoMoS/AC and $\mathrm{MoS}_{2} / \mathrm{AC}$ were prepared through the same procedures.

\section{Experimental}

All the analytical grade chemicals used in experiments were purchased from Shanghai Chemical Reagents Company (China). Synthesis of the Ni/Co-Mo sulfide NPs and their application as HYD nanocatalysts are conducted following the route illustrated in Scheme 1.

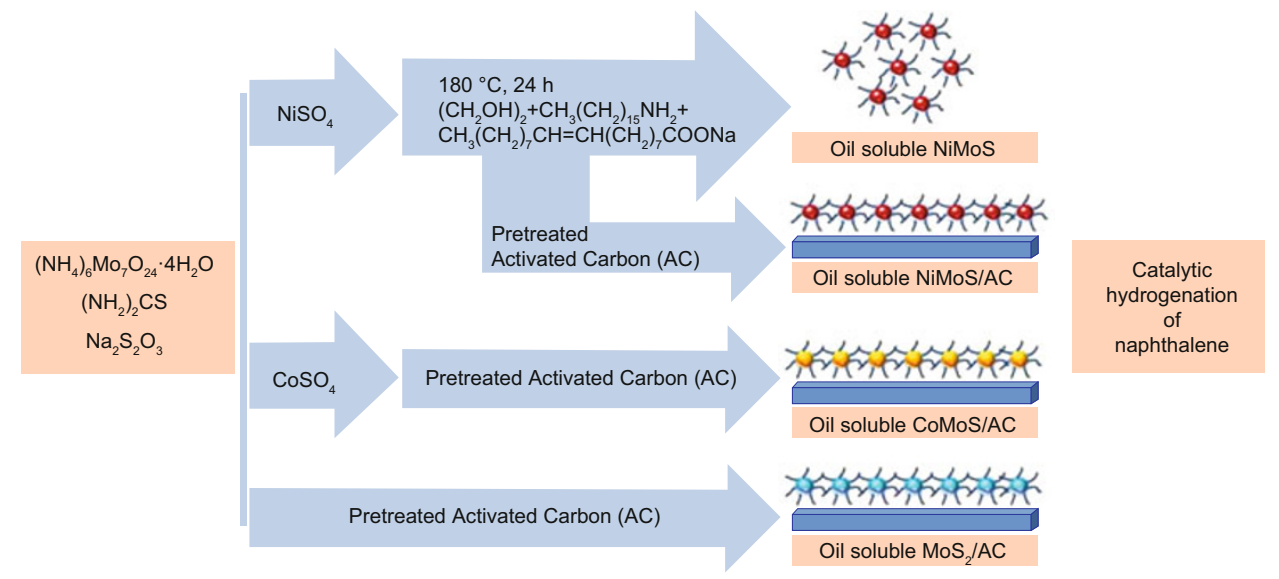

Scheme 1 Preparation of Ni/Co-Mo based sulfide nanocatalyst for hydrogenation of naphthalene

\subsection{Synthesis of oil-soluble Ni-Mo sulfide NPs}

In a typical procedure, $1.64 \mathrm{mmol}$ of nickel sulfate $\left(\mathrm{NiSO}_{4}\right), 0.37 \mathrm{mmol}$ of ammonium heptamolybdate tetrahydrate $\left(\mathrm{AHM},\left(\mathrm{NH}_{4}\right)_{6} \mathrm{Mo}_{7} \mathrm{O}_{24} \cdot 4 \mathrm{H}_{2} \mathrm{O}\right), 6.82$ mmol of thiourea $\left(\left(\mathrm{NH}_{2}\right)_{2} \mathrm{CS}\right), 1.82 \mathrm{mmol}$ of sodium thiosulfate $\left(\mathrm{Na}_{2} \mathrm{~S}_{2} \mathrm{O}_{3}\right), 1.03 \mathrm{mmol}$ of sodium oleate ( $\mathrm{SO}$, $\left.\mathrm{CH}_{3}\left(\mathrm{CH}_{2}\right)_{7} \mathrm{CH}=\mathrm{CH}\left(\mathrm{CH}_{2}\right)_{7} \mathrm{COONa}\right)$, and $0.85 \mathrm{mmol}$ of cetylamine $\left(\mathrm{CA}, \mathrm{CH}_{3}\left(\mathrm{CH}_{2}\right)_{15} \mathrm{NH}_{2}\right)$ were added together into a $50 \mathrm{~mL}$ Teflon-lined stainless steel autoclave containing $35 \mathrm{~mL}$ of ethylene glycol (EG, $\left.\mathrm{HOCH}_{2} \mathrm{CH}_{2} \mathrm{OH}\right)$. The autoclave was sealed, and heated at $180{ }^{\circ} \mathrm{C}$ for 24 hours, and then allowed to cool to room temperature. Subsequently, the precipitates were gathered through centrifugation, and then washed with distilled water and anhydrous ethanol. The precipitates were then dissolved in petroleum ether. Finally, oil-soluble NPs were collected through distillation of the filtrate, which was gained by centrifugation of the petroleum ether solution.

\subsection{Characterization of Ni-Mo sulfide NPs}

The morphology of the product (oil-soluble NPs) was examined with transmission electronic microscopy (TEM) (Hitachi H-800 transmission electronic microscope, Japan). The Fourier transform infrared (FTIR) spectra were recorded using pressed $\mathrm{KBr}$ disks with a FTIR spectrometer (Magna-750, Nicolet, USA). The ultraviolet (UV) spectra were recorded with a UV spectrophotometer (Cary-50, Varian, USA). Crystallographic information of the product was investigated with X-ray powder diffraction (XRD) using a D/Max-IIIA diffractometer (Rigaku, Japan) using graphitefiltered $\mathrm{CuK} \alpha$ radiation at a scanning rate of $2 \% \mathrm{~min}$.

\subsection{Evaluation of the catalytic performance of Ni-Mo sulfide NPs}

HYD reactions were performed with the NiMoS NPs as catalyst. These were used directly (without support) or supported on AC surfaces. To facilitate adsorption of the sulfide precursor, activation of the carbon supports was conducted as reported elsewhere (de la Puente et al, 1999; Shi et al, 2010) prior to the catalytic process, that is, 1 hour pretreatment of the carbon support in boiling deionized water and then sedimentation to remove the supernatant liquid. The above treatment was repeated twice. Then it was treated for 3 hours in concentrated $\mathrm{HNO}_{3}\left(12 \mathrm{~mol} \cdot \mathrm{L}^{-1}\right)$ at $100{ }^{\circ} \mathrm{C}$. Finally, the activated support of $\mathrm{AC}$ was obtained after vacuum drying at room temperature.

The prepared Ni-Mo sulfide NPs were first loaded onto the surface of the AC supports by the impregnation method. In a typical procedure, the AC support was impregnated with a heptane solution of the Ni-Mo sulfide NPs at $40{ }^{\circ} \mathrm{C}$ for 30 minutes, and then the $\mathrm{AC}$ supported NiMoS was heated under vacuum at $110{ }^{\circ} \mathrm{C}$ for 12 hours and calcined under $\mathrm{N}_{2}$ at 600 $800{ }^{\circ} \mathrm{C}$ for 15 minutes to obtain the NiMoS/AC catalyst. Then, the performance of the NiMoS/AC catalyst was evaluated in the catalytic HYD reactions of NAT as follows: $0.2 \mathrm{~g}$ of NAT and $0.002 \mathrm{~g}$ of the prepared nanocatalyst were dispersed in clay-refined lube base oil and put into a $50 \mathrm{~mL}$ high-pressure reactor. The reactor was purged with $\mathrm{N}_{2}$ three times and then $\mathrm{H}_{2}$ was blown into the reactor to $6.0 \mathrm{MPa}$. After 2 hours of reaction at $360{ }^{\circ} \mathrm{C}$, the reactor was cooled quickly with cold water, and the HYD product of NAT was dissolved in toluene, analyzed by gas chromatography (GC), using a Varian 3400 chromatograph (GS-Gas Pro GC/PLOT) with a flame ionization detector (FID) operating at $300{ }^{\circ} \mathrm{C}$ for 25 minutes. The other conditions for $\mathrm{GC}$ analysis was as follows: split ratio of 100:1, the feeding amount of $1 \mu \mathrm{L}$, and the temperatures of feeding entrance, initial column, and final column were 300,100 and $300{ }^{\circ} \mathrm{C}$, respectively, with a rate of temperature increase of $10.0^{\circ} \mathrm{C} / \mathrm{min}$. For comparison, 
catalysts $\mathrm{CoMoS} / \mathrm{AC}$ and $\mathrm{MoS}_{2} / \mathrm{AC}$ were also prepared through the same procedures as those for NiMoS/AC, except for the promoting metals. The mass ratio of the metal sulfide to $\mathrm{AC}$ for all the catalysts was $14 \%$.

\section{Results and discussion}

The product NiMoS consisted of well-dispersed NPs as shown in Fig. 1(a). There was a color difference between the inner part (deep color) and the outside layer (light color) of the NiMoS NPs, suggesting an inorganic-organic core-shell structure of the sulfides considering the organic species ( $\mathrm{SO}$ and CA) involved in the reaction system. The as-prepared NiMoS NPs had a narrow size distribution with an average size of about $7.6 \mathrm{~nm}$ (Fig. 1(b)). In addition, the as-prepared NiMoS NPs can form stable colloidal suspensions in several organic solvents, including heptane, dimethylformamide, and petroleum ether. The excellent lipophilicity of the Ni-Mo sulfide NPs confirmed the formation of the inorganic-organic core-shell structure with an organic layer coated at the surface of the inorganic NPs, which will contribute to their catalytic performance because of their improved oil-solubility.

The XRD patterns of the as-synthesized NiMoS NPs in Fig. 1(c) showed weak and wide diffraction peaks, indicating a low degree of crystallinity and small size of the NiMoS NPs. This result was in accord with that from the TEM images. Considering the hydrothermal conditions for the synthesis of NiMoS NPs, it can be concluded that the incorporation of $\mathrm{Ni}$ component resulted in the formation of a NiMoS phase, not the compounds of $\mathrm{NiS}_{2}$ and/or $\mathrm{MoS}_{2}$. In comparison with the standard diffraction patterns $\left(\mathrm{NiS}_{2}\right.$, JCPDS card No. 730574; $\mathrm{MoS}_{2}$, JCPDS card No. 75-1539), the most intensive (002) peak at $2 \theta=14.125^{\circ}$ for $\mathrm{MoS}_{2}$ disappeared for the asprepared NiMoS NPs, demonstrating the destruction of the long-range order of $\mathrm{MoS}_{2}$ along its $c$-axis (Huang et al, 2010; Yin and Liu, 2004; Yoosuk et al, 2010). Therefore, it can be concluded that the incorporation of promoting $\mathrm{Ni}$ atoms led to low stacking of the NiMoS NPs with more active sites, favorable to the improvement of the catalytic performance of the NiMoS NPs as HYD catalyst.

The presence of organic species on the surface of the sulfide NiMoS was further proved by FTIR (Fig. 2) and UV spectra (Fig. 3). Fig. 2 shows the characteristic stretching vibrations of the free molecules of $\mathrm{SO}$ and $\mathrm{CA}$, with signals of $\mathrm{C}=\mathrm{C}$ bond around $1,610 \mathrm{~cm}^{-1}$ and $1,480 \mathrm{~cm}^{-1}$ for $\mathrm{SO}$, and $\mathrm{N}-\mathrm{H}$ bond around $1,560 \mathrm{~cm}^{-1}$ for CA. For the oil-soluble NiMoS NPs, these absorption bands shifted to $1,740 \mathrm{~cm}^{-1}, 1,653 \mathrm{~cm}^{-1}$, and $1,460 \mathrm{~cm}^{-1}$, respectively, confirming the adsorption of the organic molecules at the surface of the sulfide NPs. The UV spectra in Fig. 3 also demonstrates the characteristic adsorption bands of SO (205 and $210 \mathrm{~nm})$, CA (205 and 231 $\mathrm{nm}$ ), and surface NiMoS NPs (220 and $235 \mathrm{~nm}$ ). The strong absorption band of oil-soluble NiMoS NPs around $200 \mathrm{~nm}$ showed $20 \mathrm{~nm}$ blue-shift as compared with its counterpart of bulk materials, and this was the result of quantum size effects. Additionally, the characteristic band of NPs at $220 \mathrm{~nm}$ also indicated the presence of surface adsorbed organic molecules. Consequently, the small particle size and low degree of crystallinity endow the NiMoS NPs with larger surface area
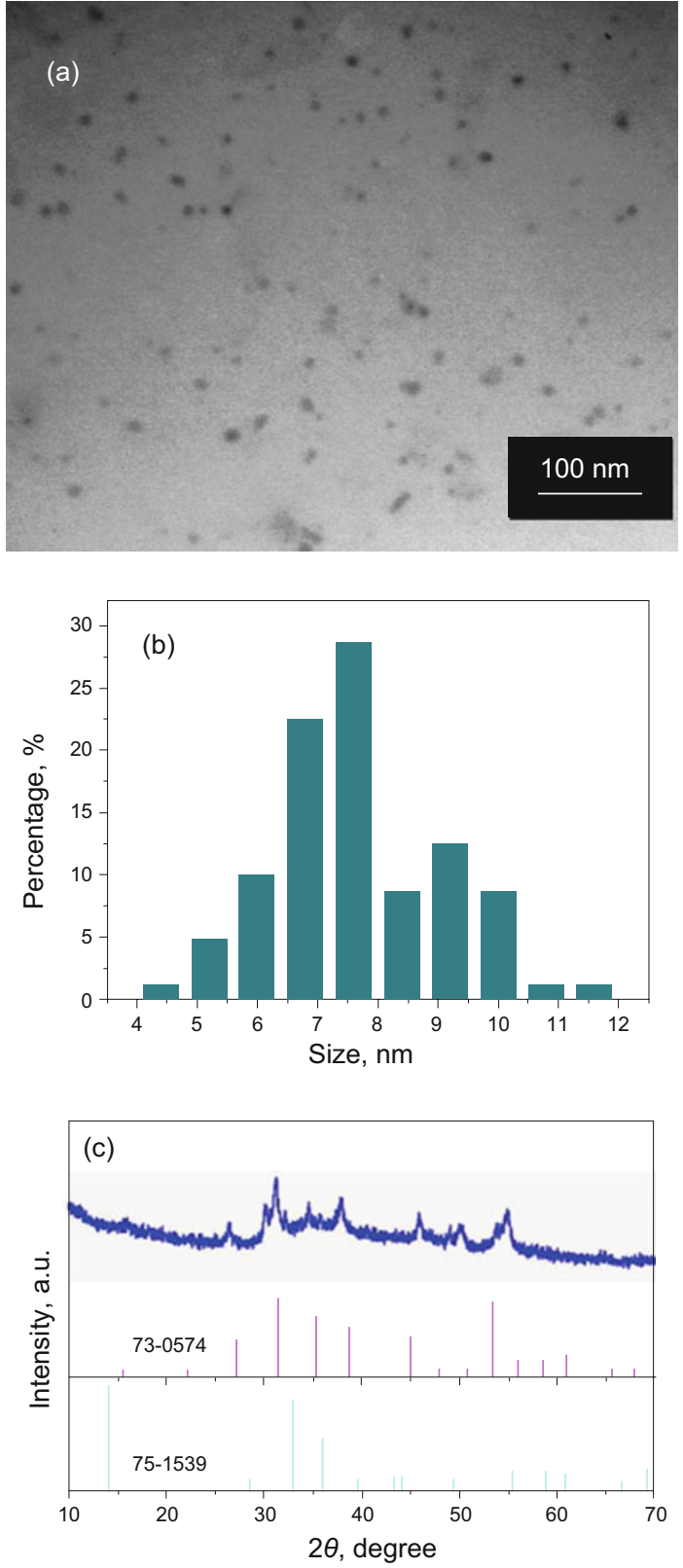

Fig. 1 TEM image (a), size distribution histograms (b), and XRD pattern (blue) (c) of the NiMoS NPs

and more active sites than CoMoS and $\mathrm{MoS}_{2}$, making the NiMoS NPs a promising candidate as a heterogeneous HYD catalyst with enhanced oil-solubility and stability.

According to the GC results, the predominant products of NAT HYD reaction were tetrahydronaphthalene $(4 \mathrm{HN})$ and decahydronaphthalene $(10 \mathrm{HN})$, instead of the ringopening or cracking derivatives through deep hydrogenation (He et al, 2013). Therefore, the catalytic hydrogenation of NAT dominated the catalytic reaction process in this work. The NiMoS NPs exhibited high activity mainly in the hydrogenation reaction instead in the ring-opening/cracking reactions. This result is accordance with that by Ferraz and coworkers using alumina-supported NiMoS catalyst (Ferraz et al, 2010).

The HYD activity of the as-prepared AC supported NiMoS nanocatalysts was measured and expressed by the 


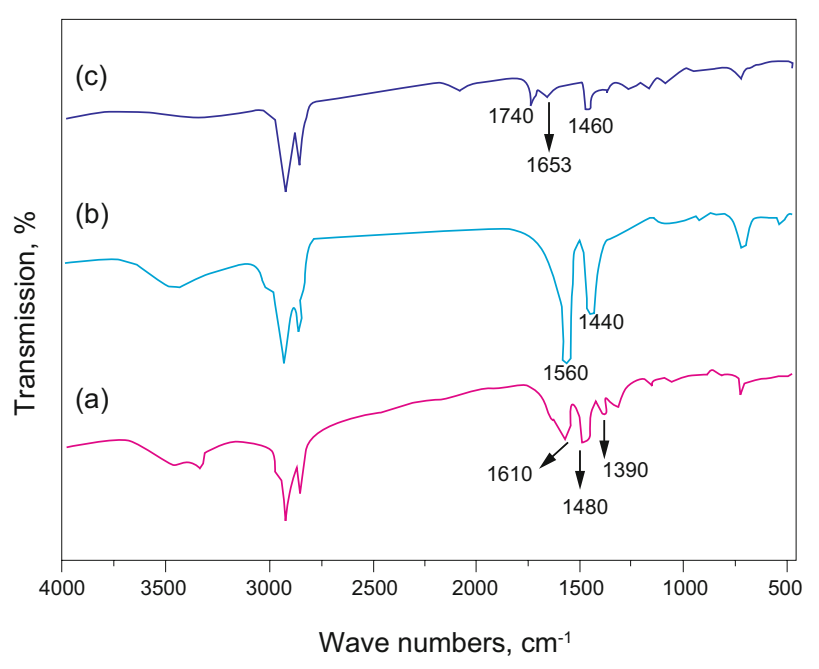

Fig. 2 FTIR spectra of (a) sodium oleate, (b) cetylamine and (c) NiMoS NPs

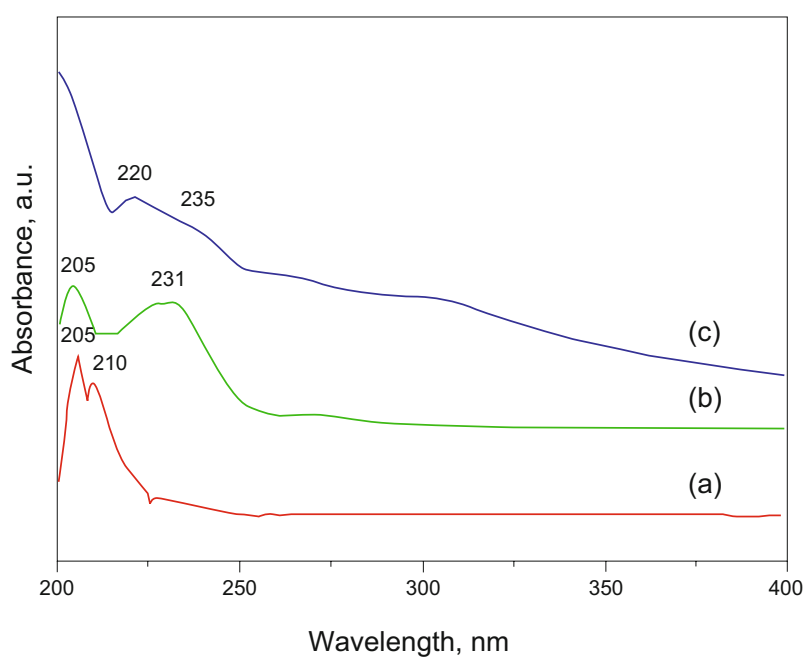

Fig. 3 UV spectra of (a) sodium oleate, (b) cetylamine and (c) NiMoS NPs

percentage of $4 \mathrm{HN}$ and $10 \mathrm{HN}$, which was calculated as the ratio of the increased GC peak area to that of the feeding NAT. Fig. 4 shows the catalytic HYD activity of NiMoS/ $\mathrm{AC}, \mathrm{CoMoS} / \mathrm{AC}$, and $\mathrm{MoS}_{2} / \mathrm{AC}$. The results indicated that the incorporation of both nickel and cobalt as promotional components enhanced the conversion of NAT as reported in other literature (Kibsgaard et al, 2010; Kumaran et al, 2006; Rodriguez et al, 1999), and the activity decreased in the order of NiMoS/AC (68\%) > CoMoS/AC (66\%) > $\mathrm{MoS}_{2} / \mathrm{AC}(53 \%)$. It has been reported that the activation and dissociation of $\mathrm{H}_{2}$ may take place through heterolytic dissociation on $\mathrm{Mo} / \mathrm{Co} /$ $\mathrm{Ni}-\mathrm{S}$ sites and homolytic dissociation near disulfide $(\mathrm{S}-\mathrm{S})^{2-}$ edge groups, respectively (de la Puente et al, 1999; Kibsgaard et al, 2009; Polz et al, 1989). Considering the same conditions for catalyst preparation and hydrogenation reaction, the improved HYD activity of NiMoS, in comparison with CoMoS and $\mathrm{MoS}_{2}$, was attributed mainly to the combined support effect and promoting effect of transition metals with diverse edge wetting effects. Furthermore, in-situ carbonization of the surface organic layer during the catalytic process will facilitate the interactions between the sulfide and the AC support. Accordingly, the sulfide-AC interactions

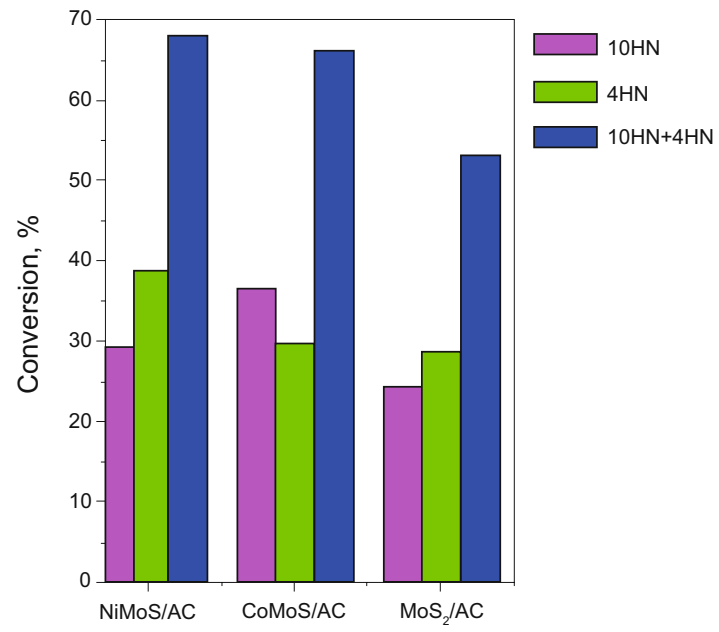

Fig. 4 Catalytic hydrogenation activity of NiMoS/AC, CoMoS/AC, and $\mathrm{MoS}_{2} / \mathrm{AC}$

became stronger and the synergic effect between the sulfide and AC support would be enhanced (Duan et al, 2007; Huang et al, 2010; Ma et al, 2001), leading to improved dispersion and reduced aggregation of the lipophilic NiMoS NPs.

To investigate the influence of $\mathrm{Ni}$ content on the conversion of NAT, the atomic ratio of $\mathrm{Ni} /(\mathrm{Ni}+\mathrm{Mo})$ was changed to tune the HYD activity of the NiMoS nanocatalyst. Fig. 5 showed that the distribution of the HYD products $(4 \mathrm{HN}, 10 \mathrm{HN}$, and their mixture) varied with the change of the $\mathrm{Ni} /(\mathrm{Ni}+\mathrm{Mo})$ ratio. It can be seen that $4 \mathrm{HN}$ reached the highest yield $(32 \%)$ at a $\mathrm{Ni} /(\mathrm{Ni}+\mathrm{Mo})$ ratio of 0.65 , while $10 \mathrm{HN}$ reached the highest yield $(48 \%)$ at a $\mathrm{Ni} /(\mathrm{Ni}+\mathrm{Mo})$ ratio of 0.35 . However, the total conversion of NAT to $4 \mathrm{HN}$ and $10 \mathrm{HN}$ decreased from $74 \%$ to $57 \%$ with an increase of the $\mathrm{Ni} /(\mathrm{Ni}+\mathrm{Mo})$ ratio from 0.20 to 0.50 . On further increase of the $\mathrm{Ni} /(\mathrm{Ni}+\mathrm{Mo})$ ratio from 0.50 to 0.65 , the conversion of NAT to $4 \mathrm{HN}$ and $10 \mathrm{HN}$ increased from $57 \%$ to $75 \%$, which may be attributed to the generation of more active NiMo-S sites and higher distribution of these active species (Mendez et al, 2013; Vit, 2007). It was confirmed that the
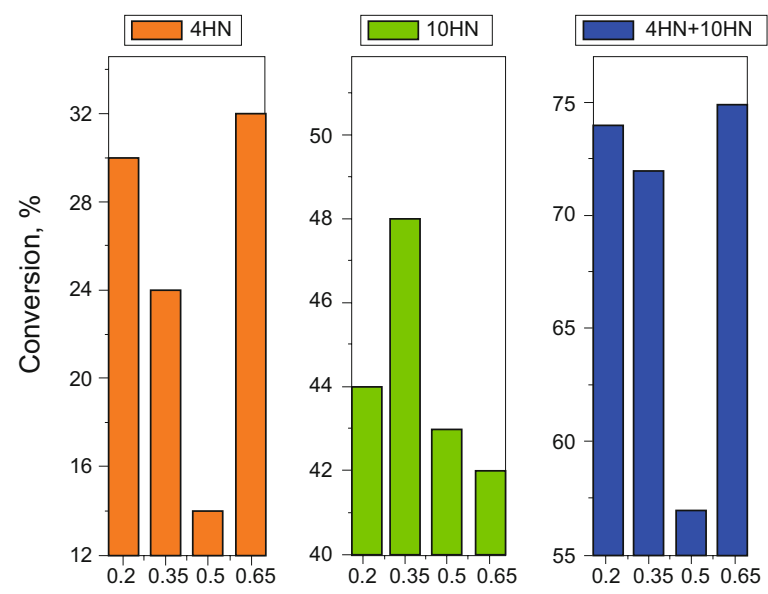

Atomic ratio of $\mathrm{Ni} /(\mathrm{Ni}+\mathrm{Mo})$

Fig. 5 Conversion of NAT at different atomic ratios of $\mathrm{Ni} /(\mathrm{Ni}+\mathrm{Mo})$ for NiMoS NPs 
HYD process depended mainly on the state of the transition metal atoms and the strength of their interaction with the supports. It is also reported that molybdenum sulfides, as strong Lewis acids, can accelerate the breaking of side chains or aliphatic chains (Cheng et al, 2006; He et al, 2011). In this work, it was found that the introduction of $\mathrm{Ni}$ in the catalyst would reduce the ratio of molybdenum and the stacking of the sulfide NPs, thus increase the ratio of Brönsted to Lewis acid sites (Liu et al, 2011b; Mendez et al, 2013). In addition, more curvatures and larger numbers of active brim sites for hydrogenation could be provided. Accordingly, structural imperfections would be formed at the sulfide edges, favorable to the adsorption of both hydrogen and NAT molecules. As a result, the generation of more Brönsted acid sites and more active edge sites would contribute to the improved HYD performance of the NiMoS NPs.

\section{Conclusions}

In summary, oil-soluble NiMoS NPs with excellent catalytic activity for NAT HYD reactions have been synthesized via a composite-surfactant-assisted-solvothermal process. The NiMoS/AC NPs favor the NAT hydrogenation instead of ring-opening/cracking processes. Activity of the as-prepared heterogeneous $\mathrm{NiMoS}$ nanocatalysts is tunable by changing the atomic ratio of $\mathrm{Ni} /(\mathrm{Ni}+\mathrm{Mo})$. Besides the improved oil solubility, the enhanced performance of NiMoS NPs is ascribed to the presence of more Brönsted acid sites and more edge sites. More detailed investigations are needed to provide insights into the catalytic mechanisms. These include controlled production of HYD catalyst with higher efficiency, more precise determination of the predominate active sites, of the anchored/free edge sites and their distributions and of the adsorption/desorption of NAT and its derivatives from the catalyst surfaces.

\section{Acknowledgements}

This work is financially supported by Shandong Provincial Natural Science Foundation (ZR2011BQ020) and the Fundamental Research Funds for the Central Universities (13CX05011A).

\section{References}

Al-Daous M A and Ali S A. Deep desulfurization of gas oil over NiMo catalysts supported on alumina-zirconia composites. Fuel. 2012. 97: 662-669

Azizi N, Ali S A, Alhooshani K, et al. Hydrotreating of light cycle oil over NiMo and CoMo catalysts with different supports. Fuel Processing Technology. 2013. 109: 172-178

Bellussi G, Rispoli G, Molinari D, et al. The role of $\mathrm{MoS}_{2}$ nano-slabs in the protection of solid cracking catalysts for the total conversion of heavy oils to good quality distillates. Catalysis Science Technology. 2013. 3: 176-182

Cheng F, Chen J and Gou X. $\mathrm{MoS}_{2}$ Ni nanocomposites as catalysts for hydrodesulfurization of thiophene and thiophene derivatives. Advanced Materials. 2006. 18: 2561-2564

Daage M and Chianelli R R. Structure-function relations in molybdenum sulfide catalysts: the "rim-edge" model. Journal of Catalysis. 1994. 149: 414-427 de la Puente G, Gil A, Pis J J, et al. Effects of support surface chemistry in hydrodeoxygenation reactions over $\mathrm{CoMo} /$ activated carbon sulfided catalysts. Langmuir. 1999. 15(18): 5800-5806

Duan A, Wan G, Zhao Z, et al. Characterization and activity of Mo supported catalysts for diesel deep hydrodesulphurization. Catalysis Today. 2007. 119: 13-18

Eijsbouts S, van den Oetelaar L C A, Louwen J N, et al. Changes of $\mathrm{MoS}_{2}$ morphology and the degree of Co segregation during the sulfidation and deactivation of commercial $\mathrm{Co}-\mathrm{Mo} / \mathrm{Al}_{2} \mathrm{O}_{3}$ hydroprocessing catalysts. Industrial \& Engineering Chemistry Research. 2007. 46(12): 3945-3954

Ferraz S G A, Zotin F M Z, Araujo L R R, et al. Influence of support acidity of NiMoS catalysts in the activity for hydrogenation and hydrocracking of tetralin. Applied Catalysis A: General. 2010. 384(12): $51-57$

He K, Zhang S, Mi J, et al. Mechanism of catalytic hydropyrolysis of sedimentary organic matter with $\mathrm{MoS}_{2}$. Petroleum Science. 2011. 8(2): 134-142

He T, Wang Y, Miao P, et al. Hydrogenation of naphthalene over noble metal supported on mesoporous zeolite in the absence and presence of sulfur. Fuel. 2013. 106: 365-371

Huang Z D, Bensch W, Lotnyk A, et al. SBA-15 as support for NiMo HDS catalysts derived from sulfur-containing molybdenum and nickel complexes: effect of activation mode. Journal of Molecular Catalysis A: Chemical. 2010. 323(1-2): 45-51

Jia M, Afanasiev P and Vrinat M. The influence of preparation method on the properties of NiMo sulfide catalysts supported on $\mathrm{ZrO}_{2}$. Applied Catalysis A: General. 2005. 278(2): 213-221

Ju X, Zhang Y, Hu Z, et al. Hydrogenation saturation discipline of 1-methyl naphthalene over Ni-Mo catalyst. Acta Petrolei Sinica (Petroleum Processing Section). 2012. 28(4): 538-543 (in Chinese)

Kibsgaard J, Clausen B S, Topsøe H, et al. Scanning tunneling microscopy studies of $\mathrm{TiO}_{2}$-supported hydrotreating catalysts: anisotropic particle shapes by edge-specific $\mathrm{MoS}_{2}$-support bonding. Journal of Catalysis. 2009. 263(1): 98-103

Kibsgaard J, Tuxen A, Knudsen K G, et al. Comparative atomic-scale analysis of promotional effects by late 3d-transition metals in $\mathrm{MoS}_{2}$ hydrotreating catalysts. Journal of Catalysis. 2010. 272(2): 195-203

Kumaran G M, Garg S, Soni K, et al. Catalytic functionalities of H- $\beta$ zeolite-supported molybdenum hydrotreating catalysts. Energy Fuels. 2006. 20(5): 1784-1790

Liu F, Xu S, Chi Y, et al. A novel alumina-activated carbon composite supported NiMo catalyst for hydrodesulfurization of dibenzothiophene. Catalysis Communications. 2011a. 12(6): 521-524

Liu L, Liu D, Liu B, et al. Relation between the morphology of $\mathrm{MoS}_{2}$ in NiMo catalyst and its selectivity for dibenzothiophene hydrodesulfurization. Journal of Fuel Chemistry and Technology. 2011b. 39(11): 838-843 (in Chinese)

$\mathrm{Lu}$ Y, Zhou W, Xia D, et al. Reaction between octene- 1 and $\mathrm{H}_{2} \mathrm{~S}$ over $\mathrm{NiMoS} / \gamma-\mathrm{Al}_{2} \mathrm{O}_{3}$ catalyst under presence of $\mathrm{H}_{2}$. Acta Petrolei Sinica (Petroleum Processing Section). 2012. 28(5): $744-749$ (in Chinese)

Ma Q, Klier K, Cheng H, et al. Interaction between catalyst and support. 2. Low coverage of $\mathrm{Co}$ and $\mathrm{Ni}$ at the alumina surface. The Journal of Physical Chemistry B. 2001. 105(11): 2212-2221

McCormick R L, King J A, King T R, et al. Influence of support on the performance of coal liquid hydrotreating catalysts. Industrial \& Engineering Chemistry Research. 1989. 28(7): 940-947

Mendez F J, Bastardo-Gonzalez E, Betancourt P, et al. NiMo/MCM41 catalysts for the hydrotreatment of polychlorinated biphenyls. Catalysis Letters. 2013. 143(1): 93-100

Polz J, Zeilinger H, Müller B, et al. Hydrogen uptake by $\mathrm{MoS}_{2}$ and sulfided alumina-supported Mo catalysts. Journal of Catalysis. 1989. 120(1): 22-28

Rodriguez J A, Dvorak J, Jirsak T, et al. Chemistry of thiophene, 
pyridine, and cyclohexylamine on $\mathrm{Ni} / \mathrm{MoS}_{x}$ and Ni/S/ Mo(110) surfaces: role of nickel in hydrodesulfurization and hydrodenitrogenation processes. The Journal of Physical Chemistry B. 1999. 103(39): 8310-8318

Shi G, Zhao Y, Huang Y a, et al. Mesoporous carbon supported Co-Mo and Ni-Mo catalysts for hydrodesulfurization. Chinese Journal of Catalysis. 2010. 31(8): 961-964

Si X, Xia D, Xiang Y, et al. Effects of reaction temperature on reactions between 1-hexene and $\mathrm{H}_{2} \mathrm{~S}$ over $\mathrm{NiMoS} / \gamma-\mathrm{Al}_{2} \mathrm{O}_{3}$. Journal of China University of Petroleum (Edition of Natural Science). 2010. 34(3): 134-139 (in Chinese)

Temel B, Tuxen A K, Kibsgaard J, et al. Atomic-scale insight into the origin of pyridine inhibition of $\mathrm{MoS}_{2}$-based hydrotreating catalysts. Journal of Catalysis. 2010. 271(2): 280-289

Vit Z. Iridium sulfide and Ir promoted Mo based catalysts. Applied Catalysis A: General. 2007. 322: 142-151

Yao S, Zheng Y, Ng S, et al. The role of nanobeta zeolite in NiMo hydrotreating catalysts. Applied Catalysis A: General. 2012. 435: 6167

Yin C and Liu C. Hydrodesulfurization of cracked naphtha over zeolitesupported Ni-Mo-S catalysts. Applied Catalysis A: General. 2004.

\section{3: $177-184$}

Yin $\mathrm{H}$, Zhou T, Liu Y, et al. $\mathrm{NiMo} / \mathrm{Al}_{2} \mathrm{O}_{3}$ catalyst containing nano-sized zeolite $\mathrm{Y}$ for deep hydrodesulfurization and hydrodenitrogenation of diesel. Journal of Natural Gas Chemistry. 2011. 20(4): 441-448

Yoosuk B, Song C, Kim J H, et al. Effects of preparation conditions in hydrothermal synthesis of highly active unsupported NiMo sulfide catalysts for simultaneous hydrodesulfurization of dibenzothiophene and 4,6-dimethyldibenzothiophene. Catalysis Today. 2010. 149(1-2): $52-61$

Zhang F, Wang Y, Zhao B, et al. Effect of different silica on the hydrodesulfurization performance of NiMo catalyst. Petrochemical Technology. 2012. 41(6): 664-670 (in Chinese)

Zhang K, Liu J, Liu Y, et al. Influence of calcination temperature on properties of Co-Mo-Ni-W/ $\gamma-\mathrm{Al}_{2} \mathrm{O}_{3}$ hydrotreating catalyst. Acta Petrolei Sinica (Petroleum Processing Section). 2013. 29(1): 28-34 (in Chinese)

Zhou Z, Chen S-L, Hua D, et al. Structure and activity of NiMo/alumina hydrodesulfurization model catalyst with ordered opal-like pores. Catalysis Communications. 2012. 19: 5-9 